\title{
Materiales didácticos basados en la historia y la epistemología de la ciencia Unidad didáctica sobre "el origen de la vida",,2
}

POR: COELHO, J." Y PRAIA, J."

Traducción: Equipo RED-CEE

Universidad Pedagógica Nacional

Revisión: Profesora Martha Herrera

Postgrado en Historia de la Educación

Universidad Pedagógica Nacional
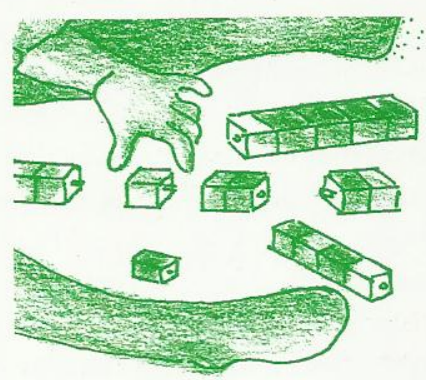

"Escuela de Secundaria Almeida Garret.

"* Departamento de Geología de la Facultad de Ciencias de la Universidad de Porto y Centro de Geología de la Universidad de Porto.
"La vitalidad de la tradición científica y su impacto positivo en la sociedad depende sobretodo de que se introduzca con éxito a los niños en la empresa, los métodos y los procesos racionales de la ciencia por profesores que comprenden y valoran la ciencia".

(Michel R. Matthews, 1994a: xv)

\section{Introducción}

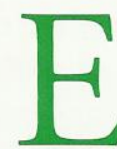

sta comunicación tiene como finalidad principal presentar materiales didácticos elaborados por el ase sor y profesores en formación en el proceso de planificación de una unidad didáctica sobre el "Origen de la Vida".

Se pretende evidenciar la contribución de la historia y la filosofía de la ciencia, abordando diferentes tesis

\footnotetext{
' El presente trabajo fue subsidiado por el Programa de Financiación Plurianual de $1 \& D(F C T)$

${ }^{2}$ Este articulo, con algunas alteraciones, fue objeto de una comunicación en el II Simposio: La Docencia de las Ciencias Experimentales en la Enseñanza Secundaria; Colegio Oficial de Biólogos, Madrid, Septiembre de 1998.
} 
plos históricos, las generalizaciones no pasarían de slogans); ॥) los episodios de la historia de la ciencia tienen una importancia incomparable en nuestra herencia cultural (Rutherford \& Ahlgren, 1995).

Para Pedrinaci (1996: 4-5) "la historia de la ciencia es ante todo, un recurso didáctico de primer orden: ¿habrá mejor forma de entender cómo se construye la ciencia que analizar algunos casos históricos? ¿Habrá mejor forma de valorizar lo que es significativo de las teorías que analizar algunas controversias históricas y reflexionar sobre la potencialidad explicativa que ofrecen diversas teorías del mismo campo del saber? iHabrá mejor forma de evitar una visión dogmática y acabada de la ciencia que analizar cómo se van sustituyendo las teorías, cómo todas las explicaciones son provisorias?".

La vertiente histórica en la enseñanza de las ciencias, frecuentemente, ha sido considerada inadecuadamente por los profesores. Algunas de estas deformaciones están presentes en las siguientes tendencias: I) considerar al pasado como un antecedente creciente y lineal del presente, sin tener en cuenta los contextos culturales de la época; ॥) evaluar la construcción de conocimiento científico de una época con los principios epistemológicos de nuestro tiempo proyectándolos para "juzgar" el pasado con los valores culturales de la sociedad occidental; III) sobrevalorar la idea de historia como resultante de la acción de "grandes hombres", en detrimento de los equipos de investigadores, olvidando la importancia de los contextos históricos, sociales y culturales; IV) desmitificar el énfasis que se hace en una historia que sólo cita los acontecimientos, ignorando los debates y los conflictos (Praia, 1996: II6); v) destacar el papel de las individualidades masculinas y si es posible del mundo occidental, desvalorizando e ignorando el carácter colectivo de la empresa científica.

\section{Los materiales didácticos}

\section{a) Las fichas de trabajo: aspectos asociados a su conceptualización.}

Las fichas de trabajo elaboradas evidencian la falibilidad de las hipótesis científicas, asumiendo el error como inherente a la propia ciencia y al progreso del conocimiento como actividad humana. En este sentido, se pretende que los alumnos "recorran" los períodos de controversia entre dos o tres hipótesis explicativas, analicen el comportamien- to de la sociedad científica y establezcan relaciones entre el progreso de la ciencia, la tecnología y la propia sociedad. Los documentos y los materiales didácticos han procurado desarrollar, a nivel del aula de clase, la problemática del Origen de la Vida según las diferentes hipótesis explicativas, teniendo en cuenta las tesis epistemológicas de Popper, Kuhn y Lakatos. El enfoque de las hipótesis explicativas, con referencias paradigmáticas, han sido también tratadas en los modelos geocronológicos y con marcos conceptuales de la Historia de la Ciencia construidos por los alumnos y concebidos por el profesor como "herramientas de trabajo" a nivel de aula.

Para este trabajo, se presentan dos fichas de un conjunto más amplio de documentos. El criterio que precedió a su selección fue el de ilustrar la articulación entre la historia y la filosofía de la ciencia.

La Ficha I (Anexo I) intentó conducir hasta el meollo del problema, en la cual se presentan dos modelos interpretativos (el exógeno ${ }^{4}$ y el endógeno ${ }^{5}$ ) para explicar cómo la vida se inició en nuestro planeta. La estrategia fue concebida para crear confrontaciones entre los varios grupos que se viniesen a constituir en defensa de un determinado modelo y la hipótesis explicativa respectiva (heterotrófica, autotrófica y cosmozóica).

La Ficha 2 (Anexo II) destacó la importancia de las arcillas, las piritas y los cuarzos como medios alternativos para el "caldo primitivo" admitido por Oparín. Estos modelos para la síntesis de compuestos orgánicos ofrecen una solución intermedia entre el mundo mineral y el orgánico en la formación de los primeros organismos vivos y son una respuesta posible a una anomalía, el agua del "caldo primitivo" no facilita reacciones de polimerización, contribuye a la solución de dos compuestos orgánicos y promueve más fácilmente la hidrólisis de las moléculas recién sintetizadas.

Parafraseando (Marques, 1995: 53) "todo esto constituye un proceso largo y continuo debido a la existencia de problemas aún no solucionados (...) y puede interpretarse de acuerdo con el falsacionismo sofisticado de Lakatos (...) en donde una teoría nunca es rechazada por la simple contrastacion de hechos contradictorios aisiados".

${ }^{4}$ Reconoce la contaminación de la tierra por moléculas orgánicas y/o por seres vivos transportados por ejemplo en los meteoritos.

${ }^{5}$ Reconoce el Origen de la Vida generado en la propia tierra. 


\section{b) Modelo didáctico ilustrativo de marcos de Historia de la Ciencia.}

A partir de material muy simple (5 cintas métricas de tela plastificada de un metro de longitud y dos soportes para material de vidrio) los alumnos seleccionaron acontecimientos relevantes de la historia de la ciencia para la comprensión de los presupuestos de cada hipótesis explicativa. La cinta I poseía una serie de episodios (descritos en etiquetas de $7 \mathrm{~cm}$ $\times 5 \mathrm{~cm}$ ) de la historia de la ciencia desde los principios de la civilización hasta el siglo XVI, las cintas 2, 3, 4 y 5 "llevaban" etiquetas con los acontecimientos de la historia de la ciencia circunscritos respectivamente en los siglos XVI, XVII, XVIII, XIX y $X X$. En términos de la escala temporal cada centímetro corresponde a un año de Historia de la Ciencia, excepto para la primera cinta en que apenas se establece una relación cronológica de los acontecimientos.

El principal objetivo de este modelo es contribuir a la comprensión de la naturaleza de la ciencia. Así mismo se han establecido algunos principios para la construcción de este modelo, con el fin de que no fuese apenas un registro "de.fechas (de hacimiento'o muerte de invęstigadores, de "eventós, obras o descubrinhientós'). . . .

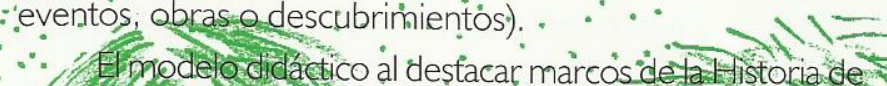

fáciles y transposiciones inmediatas, podemos afirmar que "ella ayuda a tomar conciencia de la fuerza de determinadas barreras del pasado histórico del pensamiento científico y de la forma como fueron superadas"(Santos, 1991)]; Iv) proporcionar una visión humanizada de la actividad científica (e.g. Van Helmont ( 577 - 1644) que aunque hubiese sido uno de los adeptos de la hipótesis abiogénica realizó en otros campos de la ciencia importantes investigaciones en el ámbito de los gases en el crecimiento de las plantas clorofilianas, y el Conde de Buffon publicó una importante obra "Historia Natural", en 36 volúmenes, entre 1749 y 1789 y fue uno de los defensores de la generación espontánea); v) mostrar el papel de las experiencias cruciales de la epistemología popperiana (e.g. las experiencias de Redi y de Pasteur); vi) delimitar, en términos temporales, los periodos de controversia y las revoluciones científicas en una perspectiva kunhiana; VII) evidenciar la competencia entre programas (hipótesis explicativas) regresivos y progresivos en una perspectiva lakatosiana (e.g., la hipótesis autotrófica versus hipótesis heterotrófica); vIII) mostrar las influencias de la información dentro de la propia cultura científica (e.g., las preguntas mas interesantes y determinados métodos de investigación tienen mayor probabilidad de dar.frutos; Rutherford \& Ahlgren, 1995); IX) caracterizar las relaciones entre las comunidades científicas, las relaciones políticăs y sociales de los investigadores con los organismos nstituciōnaleş y y dè gobierno.

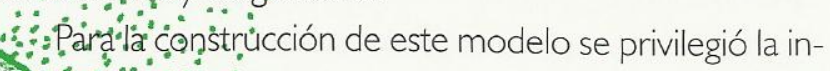

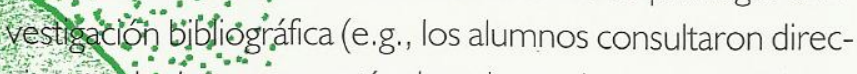
ciones de nquenet artículos de revistas, manuales y enciclopedaśmultimedia). Los alumnos también seleccionaron hechos intéseanntes a partir de los debates y de las fichas de trabajo.

\section{Incidencias diacticas}

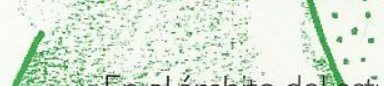

7. Fe elambito del étudiọ realizado se hace evidente que tavicacion de los haterăales en el contexto de la enseñan-

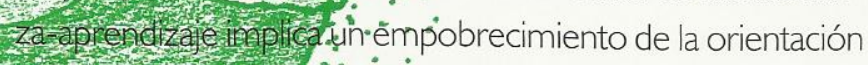
construetixsta a nyed "de là relevancia de la Historia y de la Epistenoroga de Caiencia. Este empobrecimiento se hace sentir de unartorna mís evidente en los profesores que presentanjopreocüpäciōnes más acentuadas en el área pedagógiea que en el área didáctica. 
A pesar de todo, también creemos que para utilizar las fichas de trabajo así como el modelo didáctico, los profesores deben sentir la necesidad de: I) recapitular los principios que subyacen a su construcción ; ॥) revisar, en un contexto educativo, a través de la utilización de estas "herramientas" como un polo inductor de una innovación reflexiva y más intencional.

En consecuencia, hacemos propia la metáfora de Mercé Izquierdo (1996) "la ciencia es un libro", esto es, una colección de libros y escritos susceptibles de ser reescritos en el aula para que su contenido sea más claro. También pensamos que las fichas y los modelos didácticos son un medio que proporciona una re-escritura de la ciencia gracias a la negociación de significados en el aula a través de la tarea conjunta del profesor y de los alumnos. Nonôns

\section{Referencias}

BRACK,A.(1990). Premier Signes de Vie. Science \& Vie. $\mathrm{N}^{\circ} 173$, decembre.

CACHAPUZ,A. (1992). Filosofía da Ciência e Ensino da Química:Repensar o Papel do Trabalbo Experimental. Comunicaçao ao Congreso "Las Didácticas Específicas en la Formación del Profesorado". Santiago de Compostela (Policopiado).

DUSCHL, R.A.(1997). Renovar la Enseñanza de las Ciencias. Importancia de la Teorías y su Desarrollo. Madrid: Narcea.

GAGLIARDI, R. (1988). Cómo utilizar la Historia de las Ciencias en la Enseñanza de las Ciencias. Enseñanza de las Ciencias. 6, (3). 291-296.

GIL PÉREZ, D. (1993). Contribución de la Historia y de la Filosofía de las Ciencias al Desarrollo de un Modelo de Enseñanza/Aprendizaje como Investigación. Enseñanza de las Ciencias. 11. (2). 197-212.

HODSON, D. (1986). Philosophy of Science and Science Education. Journal of Philosophy of Education. 20, (2). 215-225.

IZQUIERDO, M. (1996). Relación entre la Historia y la Filosofía de la Ciencia y la Enseñanza de las Ciencias. In Graó Educación de Serveis Pedagògics,(Ed.). Didáctica de las Ciencias Experimentales (Naturaleza e Historia de la Ciencia).8. 7-21. Barcelona:Alambique.
MARCO STIEFEL,B.(1996).Aproximación Didáctica a Textos Científicos Originales. In Graó Educación de Serveis Pedagògics, (Ed.). Didáctica de las Ciencias Experimentales (Naturaleza e Historia de la Ciencia). 8. 53-62. Barcelona:Alambique.

MARQUES, L.(1995). Teoría da Tectónica de Placas.

Contributos Relativos ao seu Percurso Histórico. In António Francisco Cachapuz, (Coord.). Formaçao de Professores. Série Cièncias $N^{o}$ 1. Aveiro: Fundaçao Joao Hacinto de Magalhaes.

MATTHEWS, M. R. (1994a). Science Teaching. The Role of History and Philosophy od Science. New York / London: Routledge.

MATTHEWS, M. R. (1994b). Historia, Filosofía y Enseñanza de las Ciencias: La Aproximación Actual. Enseñanza de las Ciencias. 12, (2). 255-277.

MOREAU, R. (1989).A Preparaçao da Vida.A Geraçao Espontànea. In Selecçoes do Reader's Digest, (Ed.)A Aventura da Vida. 32-41 e 70-71. Lisboa : Selecçoes do Reader's Digest, S.A.

PEDRINACI, E. (1996). Por unas Fructíferas Relaciones entre la Historia, la Filosofía de la Ciencia y la Educación Científica. In Graó Educación de Serveis Pedagògics, (Ed.). Didáctica de las Ciencias Experimentales (Naturaleza e Historia de la Ciencia). 8, 4-6. Barcelona: Alambique.

PRAIA, J.J.F. M.(1995). Formaçao de Professores no Ensino da Geologia:Contributos para uma Didáctica Fundamentada na Epistemologia das Ciências. O Caso da Deriva Continental. (Tese de Doutoramanto), Volume I. Universidade de Aveiro.

PRAIA,J.F(1996).Epistemología e Historia de la Ciencia:

Contribuciones a la Planificación Didáctica. La Deriva Continental. Enseñanza de las Ciencias de la Tierra. 4,(1).30-37. RUTHERFORD, F. J. \& AHLGREN,A. (1995). Ciência para

Todos. (Traduçao de C. C. Martins, do original Science for all Americans, 1995), Colecçao Aprender / Fazer Ciência. Lisoba: Livros Horizonte.

SANTOS, M.E.(1991).Mundança Conceptual na Sala de Aula. Um Desafio. Lisboa: Livros Horizonte.

TAVARES, C. \& SACARRAO, G. (1978). Curso de Biología I. Lisboa: Gabinete de Estudos e Planeamento / Ministèrio de Educaçao e Investigaçao Científica. 
A NEXO I

\section{Escuela secundaria de Almeida Garrett}

$10^{\circ}$ Año. año lectivo 96/97

Ciencias de la tierra y de la vida

Unidad: La tierra primitiva y el origen de la vida

Subunidad: El ambiente pre-biótico y el origen de la vida

Asunto: "Los presupuestos de las tres hipótesis explicativas sobre el origen de la vida" - Ficha 1

Grupo:

Nombre:

La hipótesis explicativa de la biogénesis fue verificada experimentalmente por Pasteur, cuando garantiza simultáneamente la presencia de aire ${ }^{6}$ en los balones y en un medio esterilizado de todo y de cualquier microorganismo. De esta forma las controversias sobre el origen de los seres vivos (abiogénesis versus biogénesis) parecían finalmente terminadas pero sin responder a los problemas iniciales. Con Pasteur se concluye que la vida no podía nacer de materia inerte, que la vida solo podía proceder de la vida. ¿Cómo explicar entonces la "primera de todas las apariciones"?

¿Cómo habrían surgido los primeros seres vivos en nuestro planeta en evolución (con condiciones distintas de las actuales ex., composición química de la atmósfera, cantidad de cargas eléctricas, valores de temperatura y presión diferentes a las actuales, ausencia de capa de ozono como filtro de las radiaciones ultravioleta...)?

¿Cuál es la "materia prima” necesaria para la "construcción” de los primeros seres vivos?

Prácticamente al mismo tiempo (finales del siglo xIx e inicios del xx) emergen tres hipótesis explicativas (o teorías) que pretendían dar una respuesta a los problemas formulados. La hipótesis cosmozóica o de Panspermia admitía el modelo exógeno para el inicio de la vida en la tierra, ésta consideraba posible la contaminación de nuestro planeta por moléculas orgánicas o incluso por seres vivos transportados en los cometas y meteoritos. En oposición, las hipótesis Autotrófica y Heterotrófica admitían inicialmente el modelo endógeno -la vida se pudo generar en la tierra.

La tabla 1 evidencia, para una mejor sistematización, algunos de los presupuestos iniciales de las tres hipótesis.

Los primeros seres vivos no habrían sido capaces de sintetizar las sustancias orgánicas a partir de las sustancias minerales.

Los organismos simples habrían evolucionado a lo largo del tiempo y bajo condiciones especiales hacia organismos cada vez más complejos.

Tabla I

\begin{tabular}{|c|c|c|}
\hline $\begin{array}{l}\text { Hipótesis Cosmozóica } 7 \\
\text { o de Panspermia }\end{array}$ & Hipótesis Autotrófica ${ }^{8}$ & Hipótesis Heterotrófica9 \\
\hline $\begin{array}{l}\text { - La vida no se originó en la tierra. } \\
\text { - Los microorganismos fueron } \\
\text { transportados a través del espacio por los } \\
\text { polvos movidos por las radiaciones. } \\
\text { - Los "esporas" deben haber llegado a la } \\
\text { tierra al interior de los meteoritos, } \\
\text { permaneciendo por eso protegidas de las } \\
\text { radiaciones y de otras condiciones } \\
\text { adversas. }\end{array}$ & $\begin{array}{l}\text { - Los primeros seres vivos habrían sido } \\
\text { capaces de sintetizar sustancias orgánicas } \\
\text { a partir de sustancias minerales existentes } \\
\text { en el medio. } \\
\text { - El "organismo productor" se sitúa en el } \\
\text { primer nivel trófico de las cadenas } \\
\text { alimenticias. }\end{array}$ & $\begin{array}{l}\text { - Los primeros seres vivos no habrían } \\
\text { sido capaces de sintetizar sustancias } \\
\text { orgánicas a partir de las sustancias } \\
\text { minerales. } \\
\text { - Los organismos simples habrían } \\
\text { evolucionado a lo largo del tiempo y bajo } \\
\text { condiciones especiales hacia organismos } \\
\text { cada vez más complejos. } \\
\text { - Un organismo complejo (autótrofo) no } \\
\text { habría tenido condiciones para formarse } \\
\text { en ambientes simples. }\end{array}$ \\
\hline Admite el modelo exógeno & \multicolumn{2}{|c|}{ Admite inicialmente el modelo endógeno } \\
\hline
\end{tabular}

- Una de las exigencias de los defensores de la generación espontánea era el mantenimiento del "principio activo" presente en el aire sin ser destruido por los agentes físicos (por ejemplo, calor) o por los químicos (por ejemplo ácidos).

7 Y presentada por el botánico francés P. Van Tieghem en 1884 y por el sueco Arrhenius al inicio de este siglo.
${ }^{8}$ Varios biólogos concibieron esta hipótesis a partir del trabajo sobre la fotosíntesis artificial de Melvin Calvin Premio Nóbel de Química.

${ }^{9} Y$ presentada en los años veinte de este siglo por el inglés Haldane y por el ruso Oparin. 
- Un organismo complejo (autotrófo) no habría tenido condiciones para formarse en ambientes simples.

\section{Actividades}

1. Selecciona una hipótesis explicativa (teoría) con la cual te identifiques más.

2. Forma un grupo con compañeros que también hayan escogido la misma hipótesis explicativa.

3. En tu grupo de trabajo procura defender los presupuestos de tu "teoría" y contraponer argumentos que puedan rebatir las otras "hipótesis explicativas".

\section{ANEXO II}

\section{Escuela secundaria de Almeida Garrett}

$10^{\circ}$ Año. año lectivo 96/97

Ciencias de la tierra y de la vida

Unidad: La tierra primitiva y el origen de la vida

Subunidad: El ambiente pre-biótico y el origen de la vida

Asunto: "La evolución de la hipótesis explicativa

heterotrófica" - Ficha 2

\section{Grupo:_ Nombre:}

\section{Las reacciones químicas en las zonas sub-vitales}

"Sería en el seno de este "caldo primitivo" que, en esta fase de la evolución cósmica, se da y se acelera la transformación química de la materia orgánica”.

"El agua al disolver numerosos compuestos orgánicos y minerales, favorece el encuentro entre las moléculas, aumentando las probabilidades de ocurrencia de reacciones químicas. Con todo, las moléculas orgánicas que reaccionan y aumentan de complejidad, no se ligan unas con otras por cualquier punto: poseen en su estructura química ciertos grupos de átomos que son más reactivos que otros (se denominan grupos funcionales). Un enlace entre dos moléculas resulta normalmente del "choque" entre dos grupos funcionales".

"Todavía en el "caldo primitivo", las probabilidades de encuentro eficaz serían extremadamente débiles en virtud de que los choques se darían casualmente. En los organismos vivos, por el contrario, las reacciones vitales se desarrollan en algunas fracciones de segundo, dado que las partes reactivas de las moléculas se mantienen en posiciones adecuadas hasta que se dé la reacción en presencia de catalizadores orgánicos: las enzimas. Es muy probable que en una primera fase de la evolución molecular este papel haya pertenecido a los catalizadores minerales. Ciertas su- perficies minerales o ciertos iones metálicos manifiestan, en efecto, la propiedad de fijar moléculas orgánicas y de disponerse en un orden que facilita el desencadenamiento de reacciones químicas. En estas zonas "sub-vitales", va a tener lugar una gran actividad química. Capas de arcilla, de arena o de lava, podrían haber desempeñado el papel de importantes superficies activantes".

"En 1970, A. Katchalsky ${ }^{10}$ confirmó esta hipótesis destacando el papel de ciertos tipos de arcillas, como la montmorilonita, en la condensación de aminoácidos en las cadenas polipeptídicas. Es también posible que una acción catalítica de las arcillas o de los cuarzos haya conducido, a partir de este estado, a la formación de moléculas características de la materia viva".

(Adaptado de La aventura de la vida, sus orígenes y su evolución.Selecciones de Reader's Digest de Richard Moreau)

\section{2. ¿Habrá existido un caldo primitivo?}

"Otra solución fue propuesta recientemente por G. Wächterhäuser (se trata de una verdadera revolución) -la vida se formó en medio mineral. Los primeros organismos vivos eran moléculas orgánicas simples, con carga negativa, que se desarrollaron sobre superficies de minerales ferrosos (pirita) cargados positivamente. La capa superficial orgánica fija el carbono del dióxido de carbono gaseoso, aprovechando una reacción del sulfuro de hierro con el sulfuro de hidrógeno de la atmósfera. De esta forma ocurre simultáneamente el crecimiento de las moléculas orgánicas y de la superficie del mineral".

"Esta hipótesis es seductora porque propone una solución intermedia entre el mundo mineral y el mundo orgánico, ofreciendo al químico una ocasión para ejercer su talento».

“Una vez más, la experimentación y solo ella, permitirá dar a esta hipótesis la legitimidad pre-biótica".

(Adaptado de Science Vie No 173, Dic., 1990)

\section{Actividades}

1. La evolución de los acontecimientos científicos sobre la existencia / inexistencia de un caldo primitivo parece no inviabilizar el "núcleo" de la hipótesis heterotrófica.

1.1. Presente argumentos que apunten a la anterior afirmación.

1.2. Referencie el papel de Katchalsky y de Wächterhäuser sobre el desarrollo progresivo de la hipótesis heterotrófica. NoD șs

${ }^{10}$ Cairns-Smith trabajó también con arcillas y pretendió demostrar que la síntesis ocurre y desarrolla procesos fotosintéticos rudimentarios favoreciendo la aparición de moléculas orgánicas. 